\title{
Belief Function Fusion based Self-calibration for Non-dispersive Infrared Gas Sensor
}

\author{
Yang You, Anran Xu, and Tobias J. Oechtering \\ KTH Royal Institute of Technology, Stockholm, Sweden
}

\begin{abstract}
Non-dispersive infrared gas sensing is one of the best gas measurement method for air quality monitoring. However, sensors drift over time due to sensor aging and environmental factors, which makes regular calibration necessary. In this paper, we first propose a general belief function fusion framework for NDIR gas sensor calibration, where we focus on getting a reasonable fused belief function of the true $\mathrm{CO}_{2}$ level. To deal with belief functions that may highly conflict with each other, we further propose a modified weighted average approach which utilizes the Wasserstein distance as a measure of the similarity between the belief functions. The numerical experiments show excellent initial results which confirms the belief function fusion framework for NDIR gas sensor is possible.
\end{abstract}

Index Terms-Non-dispersive infrared gas sensor, drift, self calibration, belief function fusion, Wasserstein distance.

\section{INTRODUCTION}

Gas sensors are devices used to measure the presence or concentration of gases in an area and play an important role in many applications. A non-dispersive infrared sensor (NDIR sensor) is simple spectroscopic sensor in which a nondispersive element is used to filter out the broad-band light into a narrow spectrum suitable to sense a specific gas [1] and [2]. However, NDIR sensors have been recognized to be sensitive to variations of ambient temperature, atmospheric pressure, humidity and some other environmental factors [3]. Moreover, aging of the sensor components also results in inaccuracy of the sensors. Due to this, regular calibration is needed for longterm accuracy of the sensors.

Today, state of the art of infrared gas sensor self-calibration is the well-established ABC technology (Automatic Baseline Correction) where the sensor is calibrated to a fixed value that is assumed to be the fresh air gas concentration [4]. However, this method does not work well in mega-cities where the sensors never get exposed to fresh air. Thus, designing more robust and smart self-calibration algorithms which can be widely applied in different environments becomes more and more important.

The multi-sensor information fusion [5] has become one of the most popular tools for the sensor calibration. It combines the data and information from multiple sensors to achieve more specific inferences than it could be achieved by using a single, independent sensor. In the field of multi-sensor data fusion, the belief function theory (also know as DempsterShafer (DS) theory) [6] has been widely applied since it

This research project has received funding from the European Union's Horizon 2020 research and innovation programme under grant agreement No 825272 (ULISSES). provides a particularly convenient theoretical framework for uncertainty modeling and propagation in the combination of partially reliable information. For instance, in [7], [8], the authors utilize the belief function fusion approach to achieve self-localization of mobile sensors. In [9], the belief function fusion is applied to solve the sensor fusion problem for antipersonnel mine detection. However, the traditional DS theory will result in unreasonable fused beliefs when the belief functions provided by different sensors highly conflict with each other. To mitigate this issue, pre-processing methods of the original belief functions have been proposed [10]. In [11], the authors proposed to incorporate average belief into the DS combining rule. However, it can be easily seen that simple average assigns equal weight to each body of evidence and does not consider the relationship among the evidence collected from multiple sensors, which is often unreasonable in the real application system. In this case, different weighted average approaches [12]-[14] have been proposed to management the conflicts between the belief functions and are shown to have good performance.

In this paper, we propose a general belief function fusion framework for our proposed multi-sensor information fusion problem for the calibration of NDIR $\mathrm{CO}_{2}$ sensors. We next propose a modified weighted average approach to deal with the case where belief functions highly conflict with each other. Different from the aforementioned works [12]-[14], which utilize the Jousselme distance [15] or the modified Jousselme distance as a measure of the distance between the belief functions, we propose to use the Wasserstein distance [16] as a measure of distance between the belief functions, since the Jousselme distance will not be applicable for the case where the belief functions are just simple Bayesian probability measures.

\section{System Model AND Problem Formulation}

In this section, we present a system model for our NDIR $\mathrm{CO}_{2}$ sensor network and the corresponding problem formulation for the sensor fusion. Consider an NDIR $\mathrm{CO}_{2}$ sensor system, where we have $N$ sensors which measure the timevarying $\mathrm{CO}_{2}$ level in the same environment over a certain time horizon. In this case, the $N$ sensors are regarded as measuring the same $\mathrm{CO}_{2}$ level at each time instant. According to our previous work [17], by using the hidden Markov model framework, each sensor can form its own posterior distributions of the current true $\mathrm{CO}_{2}$ level given historical measurements, i.e., the belief functions of the current true 


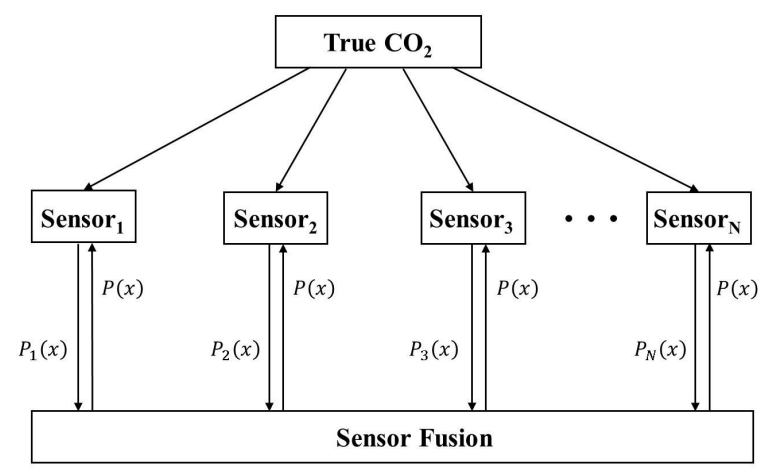

Fig. 1. System model for sensor fusion. Sensor $i$ self-awareness of measurement errors results in belief $P_{i}(x)$. The fused result $P(x)$ is later used for calibration at each sensor.

$\mathrm{CO}_{2}$ level. Denote the true $\mathrm{CO}_{2}$ level by $x \in \mathcal{X}$, where $\mathcal{X}=\left\{x_{1}, x_{2}, \ldots, x_{M}\right\}$ denotes the set of all possible sensor $\mathrm{CO}_{2}$ levels. We further use $P_{i}(x)$, which is a probability mass function, to denote the belief function of sensor $i$ on the true $\mathrm{CO}_{2}$ level. Since the sensors are unaware of the true $\mathrm{CO}_{2}$ level, to infer on the current true $\mathrm{CO}_{2}$ level, the sensors combine the information provided by each individual sensor, i.e., fuse the belief functions of each individual sensor. Finally, the fused fused belief function $P(x)$ will be adopted as new belief by all sensors (calibration). The system model depicted above is shown in Fig. 1.

\section{A. Belief Function Fusion via DS Rule}

Assume that the belief functions provided by all $N$ sensors are reliable. Our objective in this case is to design a fusion rule to combine the beliefs provided by all the $N$ sensors. The DS rule [6] provides a general framework for such a reasoning problem with uncertain information or partial information. Let $i, j \in\{1,2, \ldots, N\}$ be the index of the two sensors. Consider the most simple two sensor fusion case, where we wish to combine the belief function provided by sensor ${ }_{i}$ and sensor ${ }_{j}$. Applying the DS combination rule will lead to the following:

$$
\left(P_{i} \oplus P_{j}\right)\left(x_{k}\right)=\frac{1}{1-F} P_{i}\left(x_{k}\right) P_{j}\left(x_{k}\right), \forall x_{k} \in \mathcal{X},
$$

where $F=\sum_{x_{m}, x_{n} \in \mathcal{X}} P_{i}\left(x_{m}\right) P_{j}\left(x_{n}\right)$ denotes the normalizing factor, and ' $\oplus$, $x^{m}, x_{n}$ denotes the combination operator via DS rule.

For the $N$ sensors fusion case, the $N$-fold extension of the DS rule with singleton sets results in:

$$
\begin{aligned}
P\left(x_{k}\right) & =\left(P_{1} \oplus P_{2} \oplus \ldots \oplus P_{N}\right)\left(x_{k}\right) \\
& =\frac{1}{F^{\prime}} P_{1}\left(x_{k}\right) P_{2}\left(x_{k}\right) \ldots P_{N}\left(x_{k}\right), \forall x_{k} \in \mathcal{X},
\end{aligned}
$$

where the normalizing factor $F^{\prime}=$ $\sum_{x_{m} \in \mathcal{X}} P_{1}\left(x_{m}\right) P_{2}\left(x_{m}\right) \ldots P_{N}\left(x_{m}\right)$. The fused belief $P(x)$ is then further used by all the sensors.
B. Belief Function Fusion via Wasserstein Distance based Weighted Average

As we mentioned before, the DS rule will result in unreasonable fused beliefs when the belief functions provided by different sensors highly conflict each other, e.g., the DS rule will fail when some sensors hold highly different belief functions compared to the other sensors. To address the above issue, we propose a weighted average approach, with a weighting of each belief function based on its similarity with other belief functions. To this end, we next introduce the Wassertein distance as a measure of distance between the belief functions. The Wasserstein distance is suitable for the case where the underlying support sets of two probability measures are different. Let $Y$ and $Z$ be two random variables with probability distribution $P_{Y}$ and $P_{Z}$. The Wasserstein distance $W_{2}$ between $P_{Y}$ and $P_{Z}$ is defined as following:

$$
\begin{aligned}
& W_{2}\left(P_{Y}, P_{Z}\right)= \\
& \sqrt{P_{Y Z}: \sum_{z} P_{Y Z}=\min _{Y}, \sum_{y} P_{Y Z}=P_{Z} \sum_{y \in \mathcal{Y}, z \in \mathcal{Z}}|y-z|^{2} P_{Y Z}(y, z)} .
\end{aligned}
$$

The distance between the belief functions of sensor $_{i}$ and sensor $_{j}$ thus can be denoted by $W_{2}\left(P_{i}(x), P_{j}(x)\right), \forall i, j \in$ $\{1,2, \ldots, N\}$. After getting the distance between each pair of the sensors, we normalize all distances into the interval $[0,1]$ as follows:

$$
\hat{W}_{2}\left(P_{i}(x), P_{j}(x)\right)=\frac{2 \times W_{2}\left(P_{i}(x), P_{j}(x)\right)}{\sum_{i} \sum_{j} W_{2}\left(P_{i}(x), P_{j}(x)\right)} .
$$

With the normalized distance measure provided above, the similarity measure between belief functions $P_{i}(x)$ and $P_{j}(x)$ can be written as:

$$
S\left(P_{i}(x), P_{j}(x)\right)=1-\hat{W}_{2}\left(P_{i}(x), P_{j}(x)\right) .
$$

We further define the support degree of a given belief function $P_{i}$ as:

$$
\operatorname{Supp}\left(P_{i}(x)\right)=\sum_{j=1, j \neq i}^{N} S\left(P_{i}(x), P_{j}(x)\right),
$$

which characterizes the importance of the belief function $P_{i}$. The corresponding weighting factor of belief function $P_{i}$ is then obtained after normalization, where $\alpha_{i}=$ $\frac{\operatorname{Supp}\left(P_{i}(x)\right)}{\sum_{i=1}^{N} \operatorname{Supp}\left(P_{i}(x)\right)}$. And the weighted average of all the $N$ belief functions can be expressed $\hat{P}(x)=\sum_{i=1}^{N} \alpha_{i} P_{i}(x)$. Following [11], the fused belief function is finally obtained by using the DS rule to combine $\hat{P}(x)$ for $N-1$ times:

$$
P(x)=(\hat{P} \oplus \hat{P} \oplus \ldots \oplus \hat{P})(x),
$$

where we apply the operator ' $\oplus$ ' for $N-1$ times.

\section{NuMERICAL RESUlTS}

In this section, we present numerical results of our proposed fusion rule compared to the traditional DS rule. We use data acquired from five different NDIR gas sensors which are put in a station at highway E18 in Sweden. The sensors are 


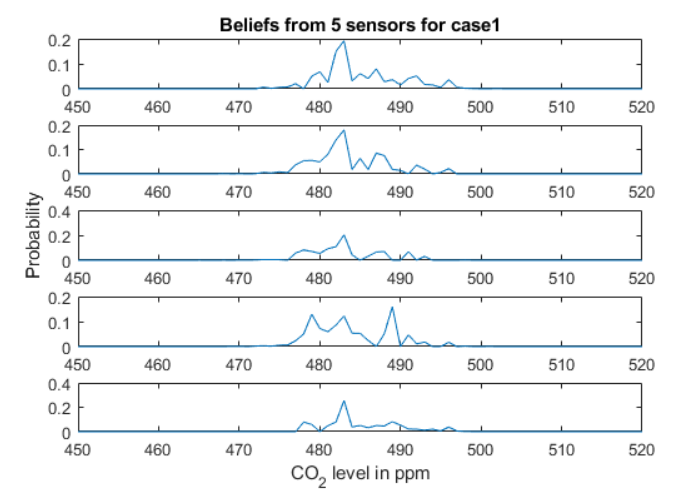

Fig. 2. Consistent belief functions provided by the sensors.
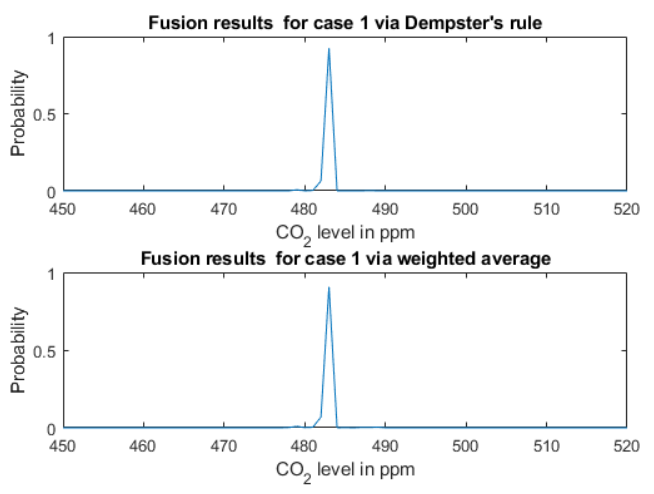

Fig. 3. Fusion results of consistent belief functions.

measuring the $\mathrm{CO}_{2}$ level in the same environment and we aim for inferring the true $\mathrm{CO}_{2}$ level based on the belief functions provided by these five sensors. As a comprehensive comparison, we show the numerical results for the following two cases.

Case 1: The belief functions provided by all sensors are consistent, no strong conflicts are present. The belief functions of all the sensors are shown in Fig. 2. By applying both Dempster's rule and our proposed fusion rule, we can get the fusion results as shown in Fig. 3. Based on Fig. 2, the true $\mathrm{CO}_{2}$ level will be most likely around 482ppm, since all five beliefs present a relatively high probability around $482 \mathrm{ppm}$. Consistently, as we can see from Fig. 3, both of the fused beliefs presents a high peak at around $482 \mathrm{ppm}$, which means the true $\mathrm{CO}_{2}$ level is most likely to be around $482 \mathrm{ppm}$. The fused beliefs are quite similar for two different fusion rules. Thus, we can conclude that both DS rule and our proposed fusion rule work well under the case where all beliefs are consistent.

Case 2: One sensor has strong conflict with the other sensors. The belief functions of all the sensors and the corresponding fusion results are presented in Fig. 4 and Fig. 5. Based on Fig. 4, we can see that the beliefs from the majority of the sensors convey the consistence message which

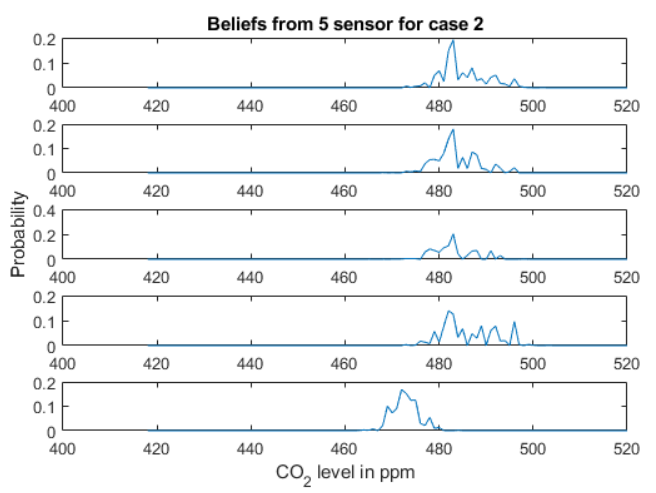

Fig. 4. Conflicting belief functions provided by the sensors.
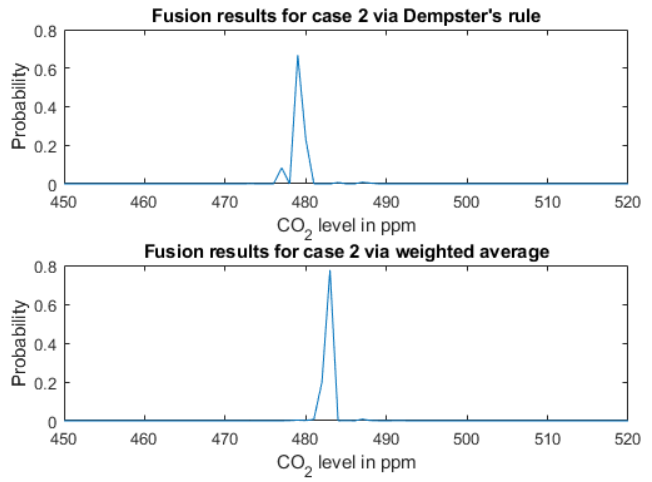

Fig. 5. Fusion results of conflicting belief functions.

again shows the true $\mathrm{CO}_{2}$ level is most likely around $482 \mathrm{ppm}$. However, the belief of the last sensor is conflicting and suggests that the true level should be some value between $470 \mathrm{ppm}$ and $480 \mathrm{ppm}$. In this case, a good fusion rule needs to mitigate the negative effect of the 'bad' evidence provided by the last sensor, and tries to reflect the evidence provided by the majority 'good' belief functions. Accordingly, the fusion results show that the DS rule fails since its fused belief suggests that the true $\mathrm{CO}_{2}$ level is most likely around $478 \mathrm{ppm}$, while the majority of the sensors have a strong belief around $482 \mathrm{ppm}$. On the other hand, our proposed fusion algorithm still has good performance since it again has an obvious peak around 482ppm.

\section{CONCLUSION}

In this paper, we studied the self-calibration of NDIR gas sensors under the framework of belief function fusion. It turns out that the general belief function fusion framework can work well in the case where no strong conflict happen and the beliefs provided by multiple sensors are consistent. A weighted average belief function fusion approach is proposed to deal with the problem when belief functions highly conflict each other. The numerical results show that for the latter case our weighted average approach has much better and reasonable fusion performance compared to the tradition DS fusion rule. 


\section{REFERENCES}

[1] J. Wong, "Ndir gas sensor," Aug. 22 1995, US Patent 5,444,249.

[2] L. Miller, Principles of Infrared Technology: A Practical Guide to the State of the Art., Springer, 2001.

[3] J. Park, H. Cho, and S. Yi, "NDIR $\mathrm{CO}_{2}$ gas sensor with improved temperature compensation," Procedia Engineering, pp. 303-306, 2010.

[4] “TN-010," Tech. Rep., SenseAir, 2000.

[5] M. Liggins II, D. Hall, and J. Llinas, Handbook of multisensor data fusion: theory and practice, CRC press, 2017.

[6] T. Denoeux and M. Masson, "Belief functions: theory and applications," in Proceedings of the 2nd international conference on belief functions. Springer, 2012, pp. 9-11.

[7] D. Alshamaa, F. Mourad-Chehade, and P. Honeine, "Tracking of mobile sensors using belief functions in indoor wireless networks," IEEE Sensors Journal, vol. 18, no. 1, pp. 310-319, 2018.

[8] D. Alshamaa, F. Mourad-Chehade, and P. Honeine, "Decentralized kernel-based localization in wireless sensor networks using belief functions," IEEE Sensors Journal, vol. 19, no. 11, pp. 4149-4159, 2019.

[9] N. Milisavljevic and I. Bloch, "Sensor fusion in anti-personnel mine detection using a two-level belief function model," IEEE Transactions on Systems, Man, and Cybernetics, Part C (Applications and Reviews), vol. 33, no. 2, pp. 269-283, 2003.

[10] E. Lefevre, O. Colot, and P. Vannoorenberghe, "Belief function combination and conflict management," Information fusion, vol. 3, no. 2, pp. 149-162, 2002.

[11] C. K. Murphy, "Combining belief functions when evidence conflicts," Decision support systems, vol. 29, no. 1, pp. 1-9, 2000.

[12] Y. Deng, W. Shi, Z. Zhu, and Q. Liu, "Combining belief functions based on distance of evidence," Decision support systems, vol. 38, no. 3, pp. 489-493, 2004.

[13] Z. Zhang, T. Liu, D. Chen, and W. Zhang, "Novel algorithm for identifying and fusing conflicting data in wireless sensor networks," Sensors, vol. 14, no. 6, pp. 9562-9581, 2014.

[14] Y. Yang and D. Han, "A new distance-based total uncertainty measure in the theory of belief functions," Knowledge-Based Systems, vol. 94, pp. 114-123, 2016.

[15] A. Jousselme, B. Grenier, and É. Bossé, "A new distance between two bodies of evidence," Information fusion, vol. 2, no. 2, pp. 91-101, 2001.

[16] C. Villani, The Wasserstein distances, pp. 93-111, Springer Berlin Heidelberg, Berlin, Heidelberg, 2009.

[17] Y. You and T. J. Oechtering, "Hidden markov model based data-driven calibration of non-dispersive infrared gas sensor," accepted at EUSIPCO 2020, available online: https://people.kth.se/ oech/esc20.pdf, 2020. 